\title{
Brain activity linked to auditory verbal hallucinations in fMRI
}

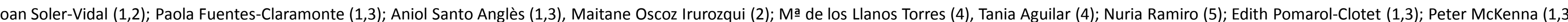

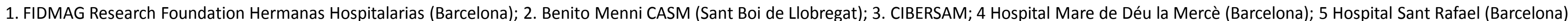

Contact: jsoler@fidmag.com

\section{BACKGROUND AND AIMS}

Auditory-verbal hallucinations (AVH) in schizophrenia have been examined in functional imaging studies (1) (2) using the 'symptom capture' technique, i.e. comparing activations when patients experience AVH to periods when they do not. Some such studies have found AVH-related activations in the auditory cortex but others have found a predominant pattern of activations outside auditory cortical areas.

The aim of this study was to examine brain activity linked to auditory verbal hallucinations in schizophrenia, and compare it to activity when hearing real auditory stimuli.

\section{MATERIALS AND METHODS}

Seven patients with schizophrenia who had nearcontinuous AVH underwent $\mathrm{FMRI}$ in a $3 \mathrm{~T}$ scanner during which they responded by button press each time they experienced AVH. During the same period they also heard, at random intervals, external spoken sentences with a similar content to their hallucinations, and responded to these with a different button press.

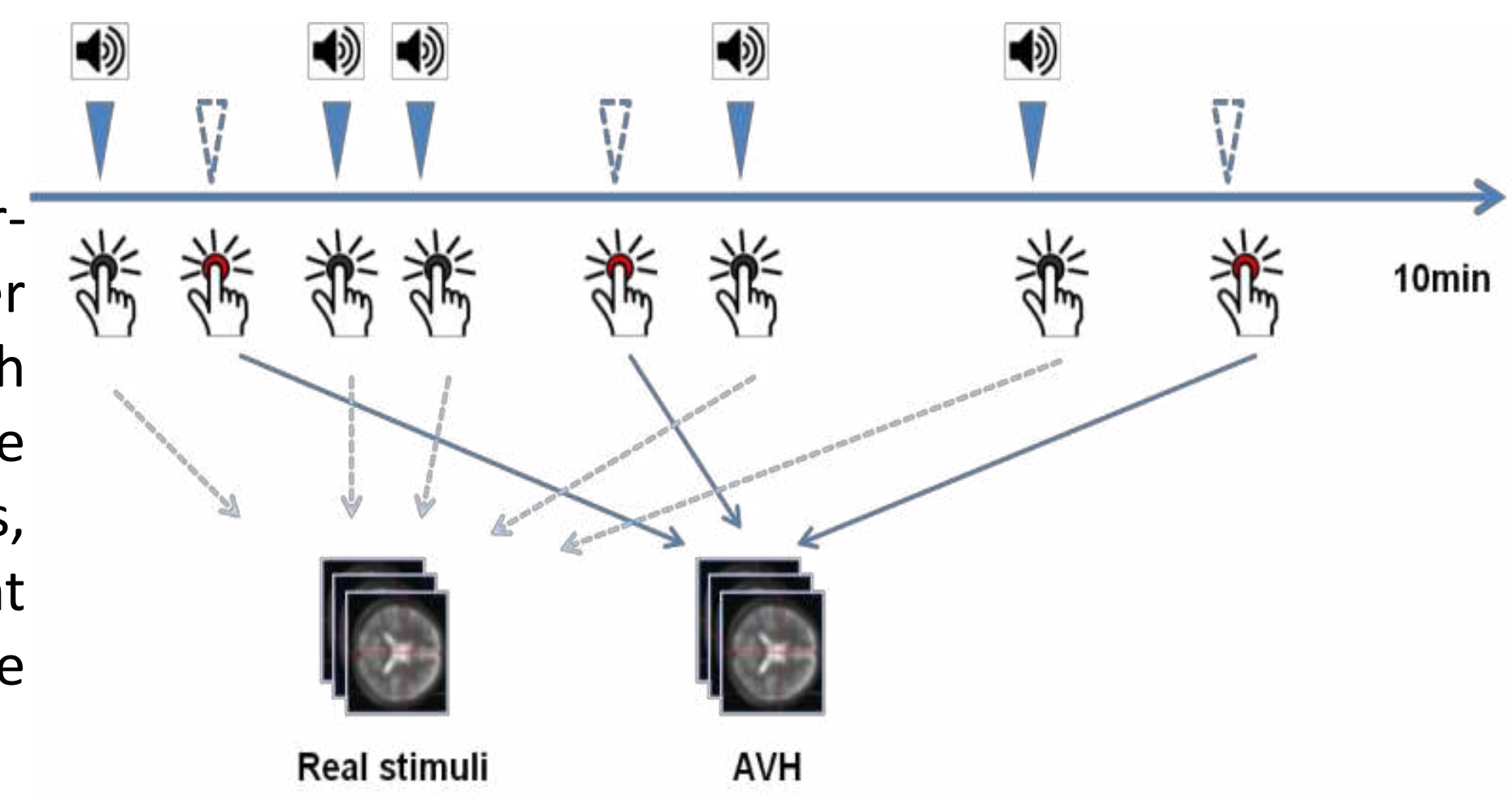

\section{RESULTS}

The real auditory stimuli activated a set of bilateral regions including in the auditory cortex, the anterior cingulate cortex and lateral frontal and parietal areas (Fig. 1). Experience of AVH activated a subset of the same areas, including the left frontal operculum, left supramarginal gyrus and the supplementary motor area. However, auditory cortical regions in the temporal lobe were not activated.

Real auditory stimuli
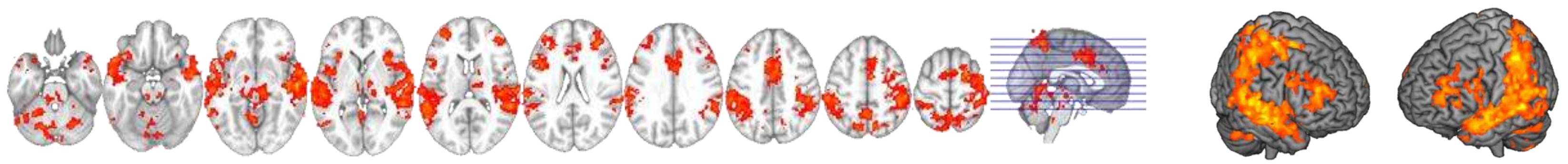

Auditory hallucinations
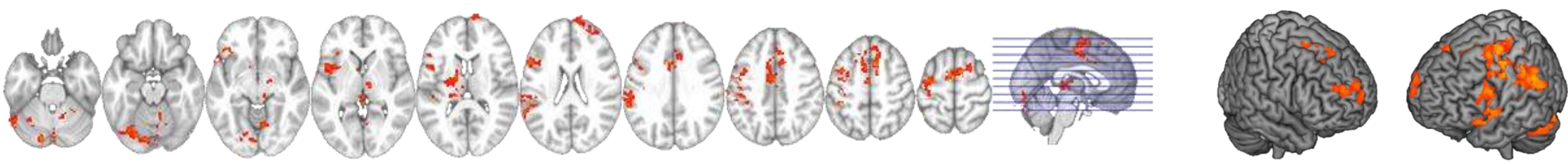

Figure 1. Brain activity in response to real auditory stimuli (top) and hallucinations (bottom). Images are shown in neurological convention (right is right).

\section{CONCLUSIONS}

Our preliminary results highlight the importance of regions involved in speech processing (Broca's area, supramarginal gyrus), among other cortical regions, in the pathophysiology of AVH. However, they do not support the proposal that $\mathrm{AVH}$ represent spontaneous abnormal activity in the auditory cortex.

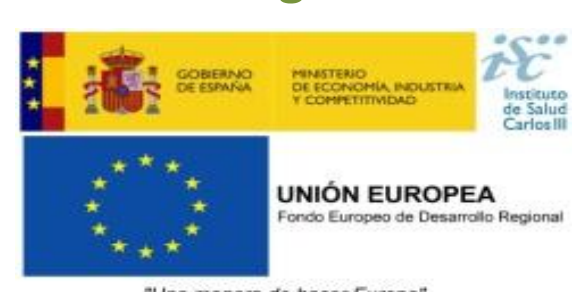

This study was funded by Instituto de Salud This study was funded by Instituto de Salud Cartos Catanya PI1800880, funded by Instituto de Salud Caject PI1800880, funded by Instituto de Salud Carlos III and co-funded by European Union (ERDF/ESF, "Investing in your future") 